\title{
Summation of the Color Processes in the Human Retina.
}

\author{
By
}

Kōiti Motokawa.

(本川弘一)

(From the Physiological Laboratory of Prof. K. Motokawa, Tohoku University, Sendai.)

(Received for publication, June 25, 1949)

The law of color mixing was established by a great number of experiments made by physicists and physiologists. The law, however, deals with physical aspects of color rather than physiological mechanisms involved in color reception. Recently, the mechanism of color reception has been elucidated to some extent by morphological, biochemical and neurological studies. Among others, the method of single fibre analysis of the optic nerve established by Hartline ${ }^{1 /}$ and Granit ${ }^{2 / 3)}$ seems to be most promising in this research field. No less serviceable is the method recently developed by the author ${ }^{45)}$ to analyse the retinal processes underlying color reception. The merit of this method is above all that it can be applied to human subjects as well as to animals.

In the present paper the effect of color mixing on the color processes in the human retina was investigated.

\section{Method.}

The electrical excitability of the eye was measured with the same method as described in the preceding papers, ${ }^{51}$ taking an electrical phosphene elicited by the electric stimulus, a constant current pulse of $100 \mathrm{msec}$. as the index. The eye generally becomes more sensitive to electrical stimulation, though temporarily, after exposure to light, and the time course of these excitability changes is characteristic of the wave-length of the light used for illumination.

A characteristic curve for each kind of light or " excitability curve" is obtained by plotting percentage increases in electrical excitability above the resting level as ordinates against the time after the end of illumination as abscissae. The illumination was always two seconds in duration and restricted to the fovea centralis of the retina.

Results.

The time course of the excitability curve depends on the wave-length 
of light, but scarcely on the intensity; for example, the excitability curve of normal man for yellow lights $(580 \mathrm{~m} \mu)$ has a maximum at 1.5 seconds, while that for blue light $(450 \mathrm{~m} \mu)$ has a maximum at about 3 seconds, as shown in (a) of Fig. 1. As the intensity of illumination is raised, the excitability curve increases in height, but remains unaltered with respect to the time to the maximum or crest time.

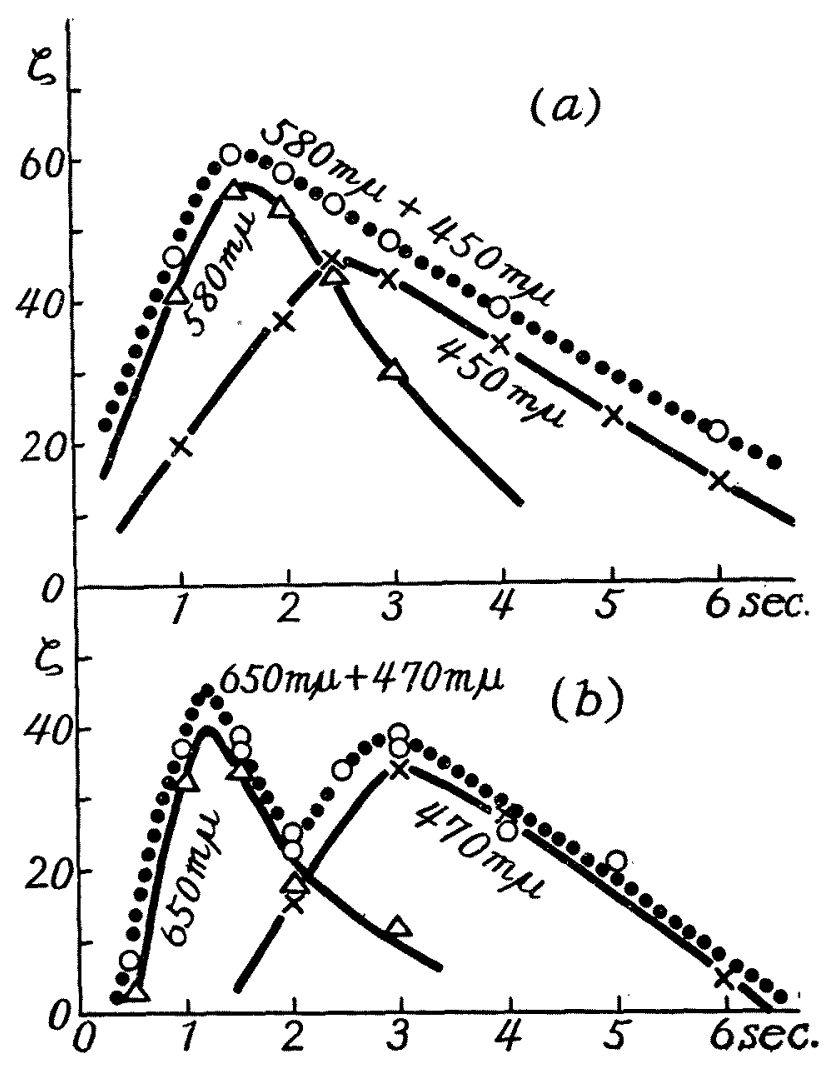

Fig. 1. Experiments of monocular color mixing. "Summation" curve: dotted. "Single" curves: drawn in full. Ordinate: $\zeta$ or percentage increase in electrical excitability of the eye. Abscissa: time after the end of two seconds' illumination of the eye.

The dotted curve in the figure was obtained by illumination with a mixture of the same colors, yellow and blue that were used separately in the experiments stated above. The curve, therefore, represents the summation of the effects of both colored lights.

As the usual experiments of color matching show, the law of additivity holds for the stimulus values of the mixed colors, whereas the present experiment indicates that this law no longer holds for the physiological quantities as measured by our method; the ordinates of the summation 
curve cannot be represented by the algebraic sum of the corresponding ordinates of the "single" curves. A similar relation can be seen in (b) of Fig. 1. In this example, red light $(650 \mathrm{~m} \mu)$ and blue light $(470 \mathrm{~m} \mu)$ were mixed, and such a summation curve as represented by a dotted curve was obtained. The single curves for both colored lights are also shown in the same figure. As can be seen from these examples, the summation curves are only slightly higher than the envelope of the single curves, and run almost parallel to it.

Next, the effect of binocular color mixing was investigated. The illumination of the eyes was carried out in the following manner. Two differently colored patches were viewed binocularly through a stereoscope so that they were fused into a single image. The left eye was exposed to a red patch $(680 \mathrm{~m} \mu)$, while the right eye was exposed to a blue patch $(470 \mathrm{~m} \mu)$. Such simultaneous illumination of the eyes caused a sensation of purple, though the quality of the color sensation sometimes fluctuated

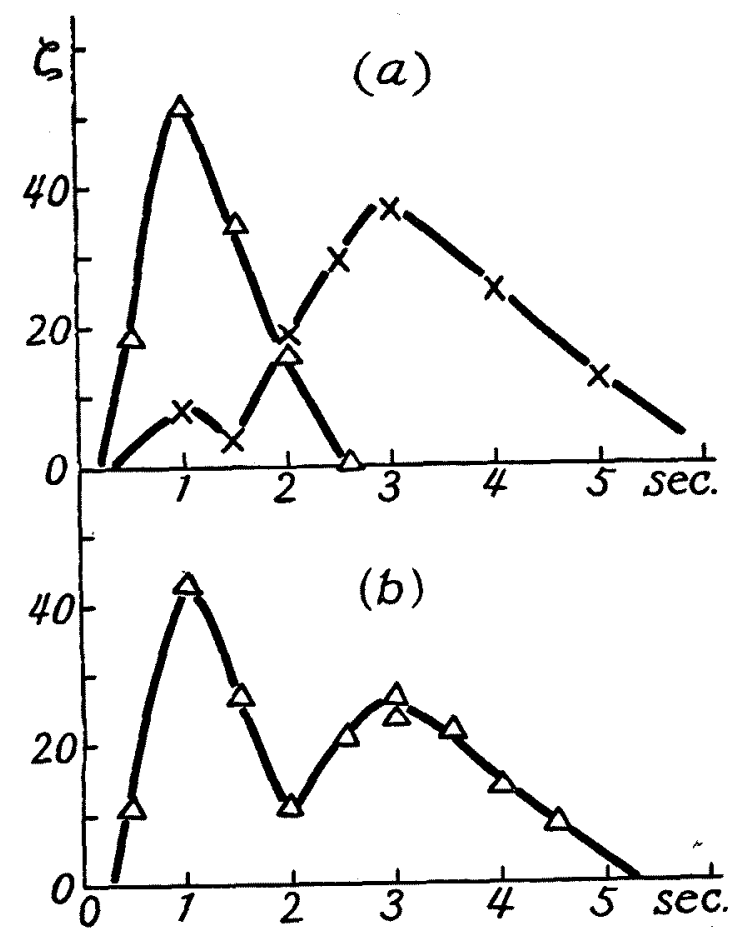

Fig. 2. Experiments of binocular color mixing. The left eye was illuminated by red light $(680 \mathrm{~m} \mu)$, while the right eye was exposed to blue light $(470 \mathrm{~m} \mu)$. The curve connecting triangles in (a) represents the excitability curve taken from the left eye, and the curve connecting crosses that obtained from right eye. The curve (b) is a control showing the effect of monocular mixing of the same red and blue lights as used in the above experiments. 
between more reddish purple and more bluish purple. The stimulating electrodes were placed in this series of experiments on the forehead slightly above the left eyebrow and on the homolateral temple to excite selectively the left eye. The data obtained with this arrangement of electrodes are illustrated as the curve connecting triangles in (a) of Fig. 2. As can be seen from this figure, the curve shows a maximum at one second, and cannot be distinguished from the excitability curve obtained by monocular illumination with red light. It is evident that, in spite of binocular fusion of red and blue light, the excitability curve taken from the left eye which was illuminated by the red light was scarcely affected by the simultaneous illumination of the right eye with the blue light.

Having rearranged the electrodes, the right eye was, in turn, stimulated electrically, and the excitability curve marked by crosses in the same figure was obtained. It shows a conspicuous maximum at three seconds, corresponding to the maximum of the curve for blue light. The much smaller elevation at one second has probably nothing to do with the simultaneous illumination of the other eye with red light, for such an accessory maximum can be observed on the usual excitability curve for blue or violet light which is capable of exciting the $\mathrm{R}$ component to a certain degree. This finding also indicates that the retinal process at an eye takes place independently of the process at the other eye. Conversely, these findings may be regarded as evidence that our phenomenon is a retinal event, or at least a physiological event occurring at a lower level of the nervous system than that required for binocular fusion of colors.

The curve represented in (b) of Fig. 1 is the result of monocular mixing of the red light with the blue light, corroborating again that summation takes place in monocular mixing, though it fails in binocular mixing of colors.

\section{Discussion.}

As pointed out already, the summation curve in monocular mixing of colors cannot be represented by algebraic summation of the single curves. This is due to the circumstance that the physiological response to an external stimulus is, in general, not proportional to the intensity of the stimulus as measured in physical units. Since the law of algebraic summation holds for color stimuli, as warranted by the law of color mixing, it is natural that the same law does not apply to the physiological effects caused by color stimuli. Instead of this law, a logarithmic law holds for the physiological effects as measured in our $\zeta$-units, percentage increases of electrical excitability after illumination; the maximum, value of $\zeta$ increases linearly with the logarithms of intensities of illumination. As re- 
ported by Motokawa and Suzuki, ${ }^{6}$ the $\zeta$-log intensity-relations for the three basic color processes indicate that the value of $\zeta$ increases by 5 to 10 on doubling the intensity of illumination. In the color mixing the stimulus value is not doubled, because different colors act on different receptors or color processes. It seems probable that peripheral summation takes place so long as the colors to be mixed involve one and the same receptor or process in the retina. If they had no receptor in common there would be no peripheral summation at all. This, however, does not imply that any summation of the impulses initiated by different peripheral receptors would not occur anywhere at higher levels of the nervous system.

As a matter of fact, the degree of summation was found to be relatively slight in the above experiments; the increase in $\zeta$ of the sammation curve above the envelope of the single curves was only 5 at the highest. If we had used a mixture of colors less different in wave-lengths, summation would have been more marked, but the increase in $\zeta$ could not be greater than 10 , the maximum value obtainable in mixing equal quantities of one and the same color.

This relation may be more easily understood, if it is analytically explained in the following manner: It may be supposed that any lightstimulus can be analysed into three components $r, g$ and $b$ which elicit the $\mathrm{R}, \mathrm{G}$ and $\mathrm{B}$ process respectively, and that, in mixing different colors, summation takes place only between components of the same kind. Let the components of a color be $r_{1}, g_{1}$ and $b_{1}$, and those of another $r_{2}, g_{2}$ and $b_{2}$, so the stimulus values of the mixture of these colors would be $r_{1}+r_{2}$ for the $\mathrm{R}$ process, $g_{1}+g_{2}$ for the $\mathrm{G}$ process and $b_{1}+b_{2}$ for the B process. From the logarithmic law stated above the three color processes elicited by these colors in separate application may be expressed in terms of stimulus values as follows:

$$
\begin{aligned}
& R_{1}=n+m \log r_{1} \\
& G_{1}=n^{\prime}+m^{\prime} \log g_{1} \\
& B_{1}=n^{\prime \prime}+m^{\prime \prime} \log b_{1} \\
& R_{2}=n+m \log r_{2} \\
& G_{2}=n^{\prime}+m^{\prime} \log g_{2} \\
& B_{2}=n^{\prime \prime}+m^{\prime \prime} \log b_{2}
\end{aligned}
$$

where $n, n^{\prime}, n^{\prime \prime}, m, m^{\prime}$ and $m^{\prime \prime}$ are constant. Since the law of color mixing states that the stimulus values are additive, the three color processes elicited by the color mixture may be expressed as follows.

$$
\begin{aligned}
& R_{1+2}=n+m \log \left(r_{1}+r_{2}\right) \\
& G_{1+2}=n^{\prime}+m^{\prime} \log \left(g_{1}+g_{2}\right) \\
& B_{1+2}=n^{\prime \prime}+m^{\prime \prime} \log \left(b_{1}+b_{2}\right)
\end{aligned}
$$

And the summation curve should be represented by the envelope of 
the component curves as given by equations (7), (8) and (9). The increase of the summation curve above the envelope of the single curves which are given by equations (1) to (6) will be obtained by subtracting the magnitude of each process of the single curve from that of the summation curve; for example, the increase in the $\mathrm{R}$ process will be equal to the difference $R_{1+2}-R_{1}=m\left\{\log \left(r_{1}+r_{2}\right)-\log r_{1}\right\}$, if $r_{1}>r_{2}$, or to the difference $R_{1+2}-R_{2}=$ $m\left\{\log \left(r_{1}+r_{2}\right)-\log r_{2}\right\}$, if $r_{2}>r_{1}$, and these differences are always smaller than $\mathrm{m} \log 2$, as can be seen from a simple calculation. Since the value of $\mathrm{m} \log 2$ as determined by the experiment of Motokawa and Suzuki lies between 5 and 10 , the increases in $\zeta$ caused by color mixing must always be smaller than this value, and this is actually the case, as shown in the above experiments.

\section{SUMMARY.}

The effect of color mixing on the retinal color processes was studied. In the first place, the excitability curves for two monochromatic lights were determined separately, and then the summation curve for the mixture of the two colored lights. From these experiments it was found that the law of additive summation which holds for the usual color mixing experiments does not apply to the physiological effects caused by lights in the retina. The summation curves were found a little higher than the envelope of the single curves obtained in the separate application of the colors, but the shape of the curve was very similar to that of the envelope.

Binocular mixing of colors had no influence upon the excitability curve; From the eye illuminated by red light was obtained an excitability curve which could not be distinguished from the excitability curve for red light, by whatever color the other eye might be illuminated simultaneously.

\section{References.}

1) Hartline, H. K., Am J. Physiol., 1938, 121, 400.

2) Granit, R. and Svaetichin, G. Uppsala Läk-Fören. förh., N. F., 1939, 45, 161.

3) Granit, R., J. Neurophysiol., 1945, 8, 195.

4) Motokawa, K., Tohoku J. Exp. Med., 1949, 51, 145.

5 ) Motokawa, K., J. Neurophysiol., 1949, 12, 291.

6) Motokawa, K. and Suzuki, K. Tohoku J. Exp. Med., 1950, 52, 195. 